\title{
A Role for the Right Anterior Temporal Lobe in Taste Quality Recognition
}

\author{
Dana M. Small, ${ }^{1}$ Marilyn Jones-Gotman, ${ }^{1}$ Robert J. Zatorre, ${ }^{1}$ Michael Petrides, ${ }^{1}$ and Alan C. Evans ${ }^{2}$ \\ ${ }^{1}$ Neuropsychology Unit, Department of Neurology and Neurosurgery, and ${ }^{2}$ McConnell Brain Imaging Center, Montreal \\ Neurological Institute, McGill University, Montreal, Quebec, Canada H3A 2B4
}

\begin{abstract}
We conducted two experiments to examine central processing of the taste of citric acid. In the first experiment, elevated citric acid recognition thresholds, but normal detection thresholds, were observed in a group of patients who had undergone a right anterior temporal lobectomy for the treatment of epilepsy, compared with a control group and a group of patients who had undergone the same operation in the left hemisphere. In the second study, using positron emission tomography, we compared regional cerebral blood flow (rCBF) in a condition in which citric acid was presented with one in which water was presented (with similar somatosensory stimulation across both conditions). We observed increased rCBF bilaterally in the caudolateral orbitofrontal cortex, in the right anteromedial temporal
\end{abstract}

lobe, and in the right caudomedial orbitofrontal cortex. The elevated recognition thresholds exhibited in patients with resection of the right anteromedial temporal lobe may be accounted for by damage in an area corresponding to that of the rCBF increase. These results suggest that although taste sensation may be computed in the primary taste cortex, recognition requires further processing by structures located in the anteromedial temporal lobe. Furthermore, they point to preferential processing of this higher-order gustatory function by the right cerebral hemisphere.

Key words: gustation; taste; amygdala; orbitofrontal cortex; insula; hedonics; positron emission tomography (PET); conditioned taste aversion (CTA)
Scott (1992) suggested that the gustatory system is organized according to the effect of the stimulus on the physiological state of the organism. For instance, it has been shown that early stages of gustatory processing may be modulated by both the internal state of the organism and the hedonic valence of a stimulus (Contreras and Frank, 1979; Jacobs et al., 1988). If this hypothesis is true, the neural circuits involved in gustatory processing should include limbic structures, which code along the novel/familiar, pleasant/ aversive, and beneficial/harmful valences.

The anterior insula/frontal operculum represents a primary gustatory area (PGA) (Pribram and Bagshaw, 1954; Benjamin and Burton, 1968; Scott et al., 1986a,b; Yaxley et al., 1990; Hirsch et al., 1994; Cerf et al., 1996; Petrides et al., 1996), and the caudolateral orbitofrontal cortex (CLOF) represents a secondary gustatory area (SGA) (Rolls et al., 1989, 1990; Baylis and Gaffan, 1991; Rolls and Baylis, 1994; Baylis et al., 1995; Small et al., 1996). Single-cell recording studies indicate that the PGA is not involved in hedonic analysis (Rolls et al., 1988; Rolls, 1993). The role of various nuclei, however, in gustatory and feeding-related processing have been described, e.g., the amygdala, which is implicated in conditioned taste aversion (CTA), gustatory neophobia, and hedonic processing (Jones and Mishkin, 1972; Rolls and Rolls, 1973; Aggleton et al., 1981; Ono et al., 1983; Borsini and Rolls, 1984; LeDoux, 1987; Dunn and Everitt, 1988; Yamamoto et al., 1994).

In the traditional view of sensory organization, the primary

Received March 3, 1997; revised April 9, 1997; accepted April 11, 1997.

Funding was provided in part by Grants MT 10314 and SP-30 from the Medical Research Council of Canada, and by the McDonnell-Pew Cognitive Neuroscience Center. We thank the technical staff of the McConnell Brain Imaging Unit and of the Medical Cyclotron for their invaluable assistance.

Correspondence should be addressed to Dana Small, Neuropsychology Unit, Department of Neurology and Neurosurgery, Montreal Neurological Institute and Hospital, 3801 rue University, Montreal, Quebec, Canada H3A 2B4.

Copyright (C) 1997 Society for Neuroscience $0270-6474 / 97 / 175136-07 \$ 05.00 / 0$ cortical area denotes the first cortical representation of a sensory stimulus, where detection and sensation occur, whereas recognition of the stimulus is a function ascribed to secondary cortical areas. There is evidence for a dissociation between areas involved with sensation and areas involved with recognition of gustatory stimuli (Kluver and Bucy, 1938; Blum et al., 1950; Pribram and Bagshaw, 1954; Henkin et al., 1977). Studies examining this dissociation implicate the anterior temporal lobe (ATL) in taste recognition. Lesions of the ATL have been associated with raised recognition thresholds in humans (Henkin et al., 1977) and dietary changes in nonhuman primates (Kluver and Bucy, 1938; Blum et al., 1950; Pribram and Bagshaw, 1954), suggesting limbic involvement in taste quality analysis.

To examine the possibility of a dissociation between gustatory sensation and perception that may be accounted for by integration of the gustatory code with limbic aspects of feeding, we designed two experiments. The first compared citric acid detection thresholds (DThs) and recognition thresholds (RThs) in healthy volunteers and in patients with excision from either the left ATL (LT) or the right ATL (RT) for surgical treatment of intractable epilepsy. We predicted RTh deficits in patients with ATL excision, reflecting the importance of this region in processing taste quality, but no impairments on DTh, because the PGA was intact.

In the second experiment, positron emission tomography (PET) was performed with healthy volunteer subjects to assess brain activation during presentation of a citric acid solution compared with a baseline condition.

\section{EXPERIMENT 1}

Subjects. Subjects were 21 patients at the Montreal Neurological Hospital who had undergone unilateral resection from the ATL for the treatment of pharmacologically intractable epilepsy. All patients had epilepsy arising from a single focus, determined by clinical pattern, electroencephalographic recordings, and magnetic resonance imaging (MRI) scans. 

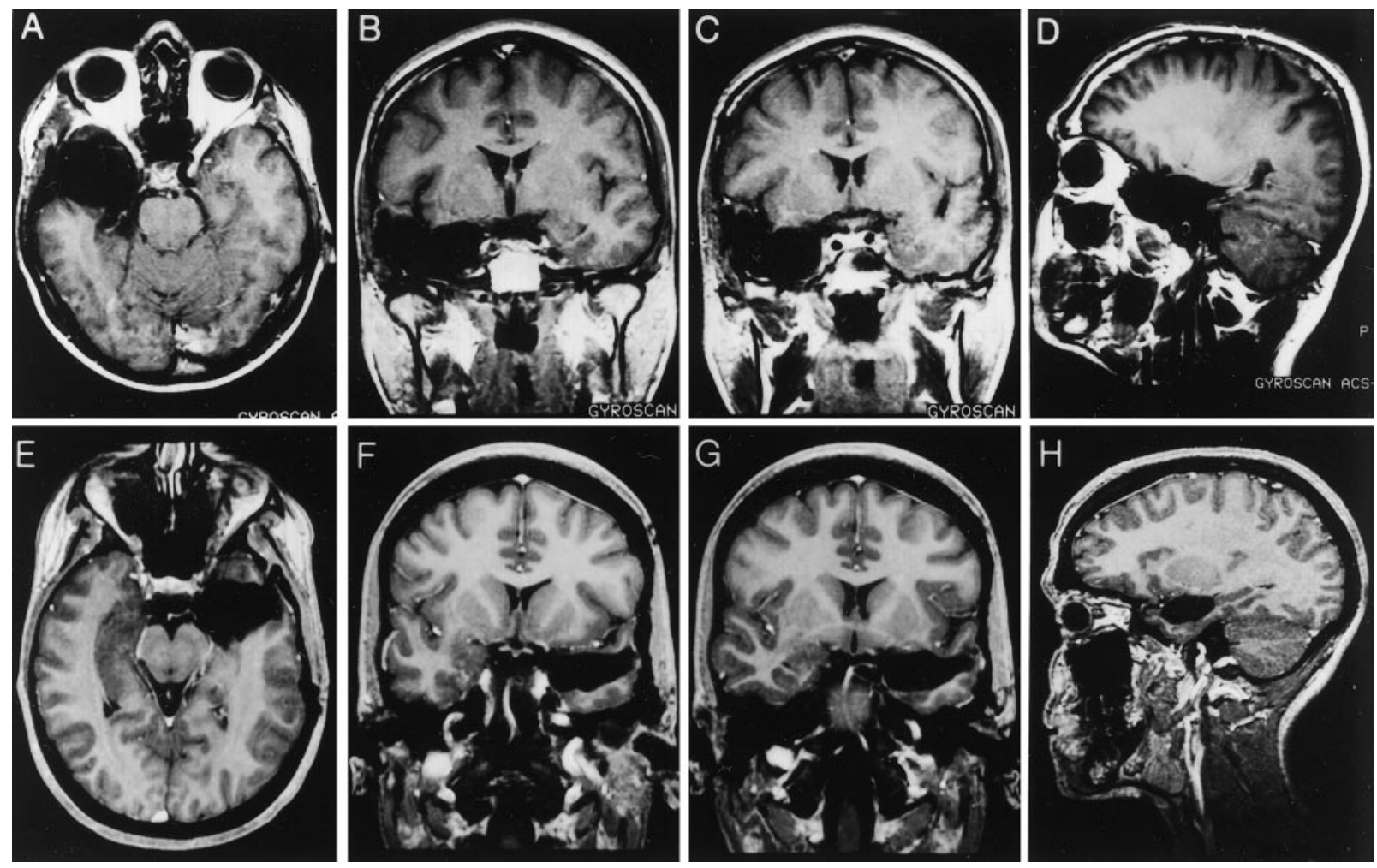

Figure 1. MRI scans illustrating representative resections. Top row, Slices from the postoperative MRI of a patient from the LT group. Bottom row, Slices from the postoperative MRI of a patient from the RT group. $A, E$, Horizontal; $B, F$, coronal; $C, G$, coronal; $D, H$, sagittal. Slices were selected to provide the optimal view of amygdaloid removal. The resections presented here are representative of all patients in this study and are typical of surgeries performed at the Montreal Neurological Institute for the relief of intractable temporal lobe epilepsy.

According to surgical reports, all patients had had at least four fifths of the amygdala and uncus removed as well as partial resection of the hippocampus ranging in length from 1.5 to $4 \mathrm{~cm}$. Varying amounts of the parahippocampal gyrus had also been removed, ranging in length from 0 to $5 \mathrm{~cm}$. In addition, 13 of the 21 patients had had removal of temporal neocortex $(\mathrm{RT}=8$ of $12 ; \mathrm{LT}=5$ of 9$)$. In these patients, the neocortical resections ranged between 4 and $6 \mathrm{~cm}$ along the first and third temporal gyri, with $3 \mathrm{~cm}$ resections in the second temporal gyrus. One patient in the $\mathrm{RT}$ group had an additional removal of the cortex adjacent to the middle cerebral artery, including partial resection of the insular cortex. Because the amygdala was the structure of interest in this study, the extent of amygdaloid resection was evaluated in postoperative MRI scans by an expert in volumetric MRI measurement (Fig. 1). It was confirmed that in all cases there had been radical excision of the amygdala comprising at least four fifths of the total volume.

All patients were of normal intelligence, with a Full-Scale Wechsler IQ rating (Wechsler, 1981) of at least 75, and were left-dominant for language function as determined by neuropsychological testing. A group of 15 healthy volunteers, roughly matched for age, sex, and smoking habits, were also tested and constituted the control group (Table 1).

Materials. UPS grade citric acid was mixed with double-distilled deionized water to make solutions ranging in concentration from $1.0 \times 10^{-2} \mathrm{M}$ to $1.0 \times 10^{-7} \mathrm{M}$. Citric acid was chosen for two reasons: (1) to reduce individual differences in detection attributable to diet (i.e., people who use more salt are less sensitive to salt), and (2) because Henkin et al. (1977) observed the most significant impairment with their sour stimulus when they assessed DThs and RThs in patients with ATL removals. All solutions were stored in glass test tubes at room temperature and replaced every 2 weeks. Solutions were presented to subjects in $10 \mathrm{ml}$ disposable plastic beakers, which were washed and recycled.

Procedure. A modified staircase method was used to establish DTh (Doty et al., 1984). On each trial (beginning at $1.0 \times 10^{-6} \mathrm{M}$ ), two cups containing liquid were presented. One contained water plus citric acid and the other just water. Subjects sipped both cups, rinsing after each with double-distilled deionized water, and then indicated which cup contained a taste other than water. If the response was incorrect, on the next trial a higher concentration of the citric acid solution was presented.
Table 1. Subjects

\begin{tabular}{lcllll} 
Group & $n$ & $\begin{array}{l}\text { Mean IQ } \\
(\text { range })\end{array}$ & $\begin{array}{l}\text { Sex } \\
(\mathrm{W}, \mathrm{M})\end{array}$ & $\begin{array}{l}\text { Mean age } \\
(\text { range })\end{array}$ & $\begin{array}{l}\text { Smokers } \\
(n)\end{array}$ \\
\hline LT & 9 & $\begin{array}{l}100 \\
(82-120)\end{array}$ & 3,6 & 34 & 3 \\
& & & $(17-52)$ & \\
RT & 12 & $\begin{array}{l}99 \\
(78-128)\end{array}$ & 8,4 & $\begin{array}{l}37 \\
(22-56)\end{array}$ & 2 \\
& & & 4,11 & $\begin{array}{l}30 \\
(22-41)\end{array}$ & 4
\end{tabular}

*IQ testing was not performed on control subjects. LT, Left temporal; RT, right temporal; C, control.

If the response was correct, the same concentration was presented a second time. If the subject responded correctly a second time, the concentration of citric acid solution was lowered for the next trial. A change in direction from increasing concentrations to lowering them, or vice versa, constituted a reversal. Seven reversals were obtained to complete the test. Concentrations for the last four trials were averaged to determine the DTh. Subjects were told that if at any time they knew what taste they were sipping they should inform the experimenter; however, no feedback was given until the end of testing.

Once the DTh was determined, subjects were asked whether they could identify the taste they had been sipping. If they did or if they had correctly identified the taste during the DTh testing, the concentration at which they informed the experimenter of the taste quality was taken as their RTh. Six control subjects and five patients (four LT and one RT) correctly identified the tastant in this way. All other subjects were given cups of increasing concentration until they could recognize the taste. The highest concentration given during the DTh examination was used as the starting point. Various responses were considered correct as long as they resembled a sour drink or food (i.e., "sour," "grapefruit," "lemon") (Table 2). The concentration at which each subject gave a correct response was taken as the RTh. 


\begin{tabular}{llll}
\hline \multicolumn{4}{l}{ Table 2. Responses taken as recognition of the tastant citric acid } \\
Response & LT & RT & C \\
\hline Sour & 3 & 5 & 4 \\
Lemon & 1 & 2 & 3 \\
Citrus & 2 & 2 & 0 \\
Acidic & 0 & 0 & 3 \\
Baking soda & 0 & 0 & 2 \\
Grapefruit & 1 & 0 & 1 \\
Vinegar & 1 & 1 & 0 \\
Apple & 0 & 1 & 0 \\
Tangy & 1 & 0 & 0 \\
\hline
\end{tabular}

LT, Left temporal; RT, right temporal; C, control.

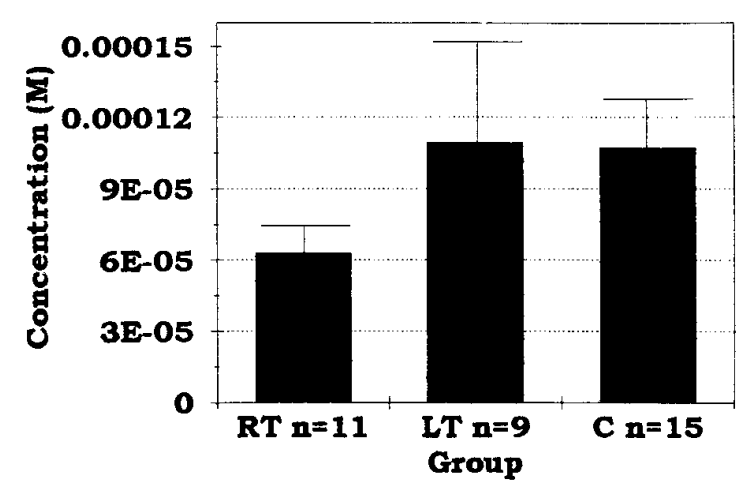

Figure 2. Mean detection thresholds for citric acid in patients with resection from left or right temporal lobe and healthy control subjects. $L T$, Left temporal; $R T$, right temporal; $C$, control.

\section{RESULTS \\ DThs}

An $\alpha$ level of 0.05 was used for all statistical tests. A one-way analysis of covariance (ANCOVA) was performed to determine whether there was a difference between groups in DTh. Sex, age, and smoking habits served as covariates. No significant differences between the groups were observed $\left(F_{(2,28)}=0.66 ; p=0.52\right)$ (Fig. 2).

We plotted individual data points for DThs and identified an outlier with a DTh of $1.4 \times 10^{-3} \mathrm{M}, 5 \mathrm{SD}$ above the overall mean threshold of $1.3 \times 10^{-4} \mathrm{M}\left(\mathrm{SD}\right.$ of $\left.2.4 \times 10^{-4} \mathrm{M}\right)$ (Fig. 3). Therefore, the data for this patient were excluded from the DTh analysis. Interestingly, this patient had had an earlier surgery in 1979, at which time parts of insular cortex were commonly excised. Analysis of the MRI scan showed damage of the ventralmost part of the insular cortex [agranular insular cortex (AIC)] adjacent to the middle cerebral artery (Fig. 4).

\section{RThs}

A one-way ANCOVA was performed to assess possible differences between groups on the basis of RTh. Sex, age, and smoking habits served as the covariates. This analysis yielded a significant difference between the groups $\left(F_{(2,28)}=11.83 ; p=0.00\right)$ (Fig. 5). This result was pursued with a Tukey HSD, which revealed that the RT group was significantly impaired compared with both the LT group $(p=0.02)$ and the control group $(p=0.02)$ (Fig. 5).

\section{Age, gender, and smoking habits}

The relative contributions of age, gender, and smoking habits were examined by a casewise regression analysis. No contribution of these variables on between-group differences was found.

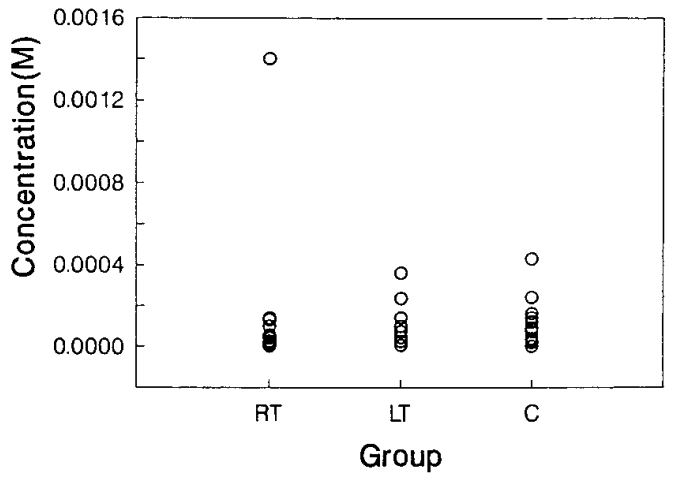

Figure 3. Distribution of detection thresholds within and across groups. Note the outlier, whose threshold is $5 \mathrm{SDs}$ higher than all other subjects. $L T$, Left temporal $(n=12) ; R T$, right temporal $(n=9) ; C$, control $(n=15)$.

\section{EXPERIMENT 2}

Subjects and materials. Ten healthy right-handed volunteers, five men and five women ranging in age from 22-41 years, underwent functional scanning with PET and MRI anatomical scanning. All subjects reported having a normal ability to taste. A gustatory test was conducted before scanning to screen for gross unsuspected gustatory deficits; none were found. The study was approved by the Ethics Committee of the Montreal Neurological Hospital, and all subjects gave informed consent.

UPS grade citric acid was dissolved in double-distilled deionized water to make a $0.023 \mathrm{M}$ solution. Tongue-shaped filter papers, made from coffee filters, were soaked either in this solution or in double-distilled deionized water for $\sim 5$ min before presentation. During scanning, the papers were placed gently on the subject's tongue with metal tweezers.

Procedure. Subjects were instructed not to eat or drink anything for at least $2 \mathrm{hr}$ before PET scanning. Two $60 \mathrm{sec}$ PET scans were conducted. In the first, five filter papers soaked in double-distilled deionized water were presented consecutively with metal tweezers to subjects lying in the PET scanner. Subjects were instructed to open their mouths when they saw the stimulus approaching. The filter paper was then placed in the mouth and left there for $\sim 7 \mathrm{sec}$, after which it was replaced with a fresh one. During the second scan, four filter papers soaked in $0.023 \mathrm{~m}$ citric acid solution were presented consecutively, followed by one filter paper soaked in water. Subjects were told that they would receive filter papers with or without a tastant and to indicate the presence or absence of a tastant by pressing either a left or right mouse button. Before scanning, all subjects underwent a pretest to familiarize them with the procedure.

PET scans were obtained with a Scanditronix PC-2048B system, reconstructed with a $20 \mathrm{~mm}$ Hanning filter (Evans et al., 1991a). Regional cerebral blood flow (rCBF) in experimental and control conditions was measured using the water bolus $\mathrm{H}_{2}{ }^{15} \mathrm{O}$ methodology (Raichle et al., 1983). MRI scans (160 slices; $1 \mathrm{~mm}$ thick) were obtained with a Philips ACS III system (1.5 T). MRI volumes were co-registered with the PET data (Evans et al., 1991b), and each matched MRI/PET data set was linearly resampled into a standardized stereotaxic coordinate system (Talairach and Tournoux, 1988; Evans et al., 1991b). A $t$ statistic for condition-dependent change in $\mathrm{rCBF}$ of each three-dimensional voxel was created by dividing each voxel by the SD in rCBF (pooled across all intracerebral voxels) (Worsley et al., 1992). Average MRI and $t$ statistic volumes were merged to localize $t$ statistic peaks (Evans et al., 1992). The statistical significance of focal rCBF changes was assessed using threedimensional Gaussian random field theory (Worsley et al., 1992). For an exploratory search involving all peaks within the gray matter volume of $600 \mathrm{ml}$, the threshold for reporting a peak as significant was set at $t=3.5$, corresponding to an uncorrected probability of $p<0.0002$ and a multiple comparison-corrected false-positive rate of 0.58 per volume. For predicted $\mathrm{rCBF}$ changes in specific brain areas, the threshold for significance was set at $t=3.00$, corresponding to $p<0.0013$.

\section{RESULTS}

Subtraction of CBF in the baseline condition from that in the citric acid condition was performed to isolate rCBF increases caused specifically by citric acid stimulation (Table 3 ). No differ- 


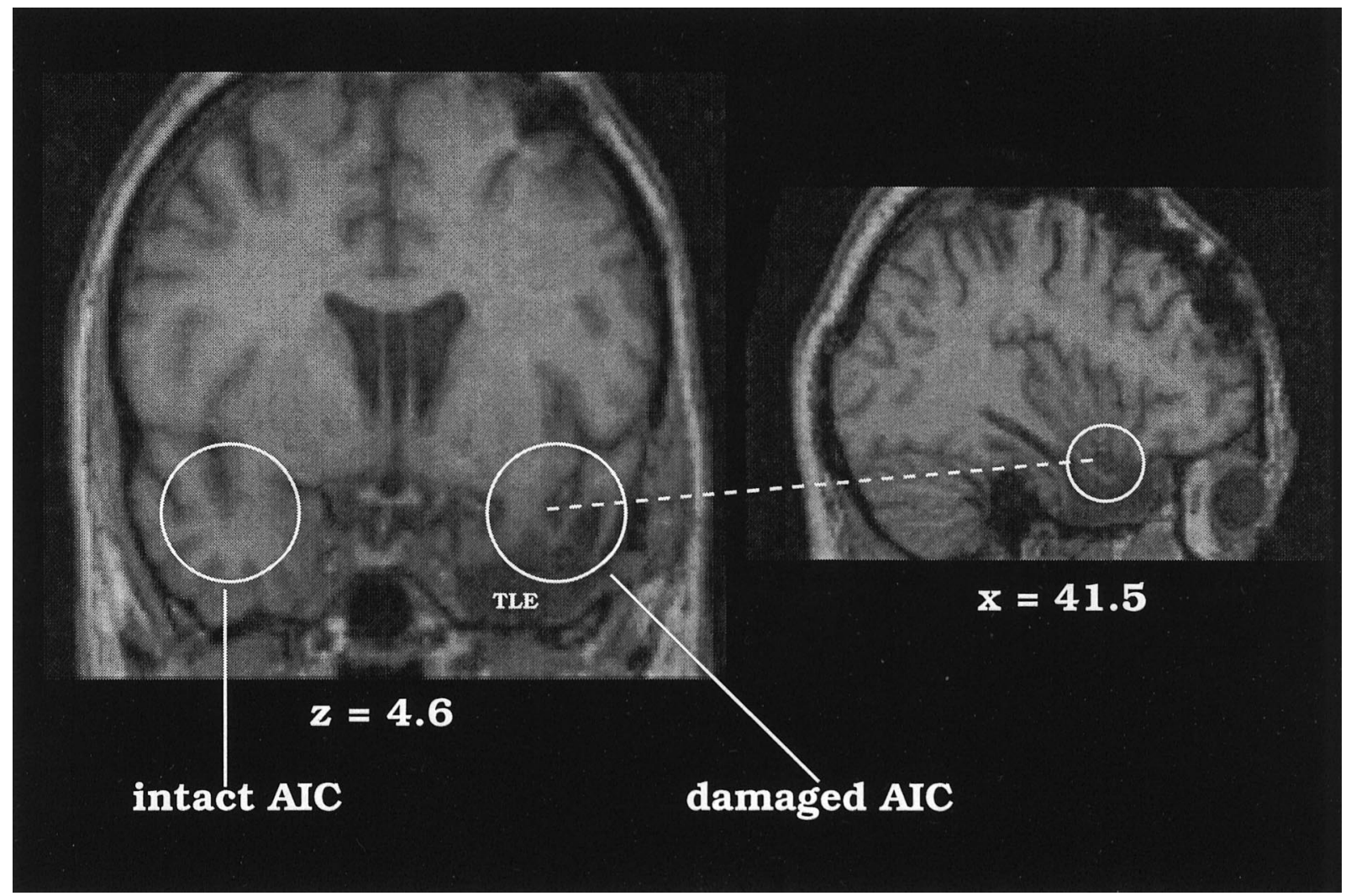

Figure 4. Resection of anterior insular cortex in the outlier. Left, Coronal section from the postoperative MRI of the subject whose detection threshold was also impaired. Note the healthy left insular cortex compared with the damaged agranular insular cortex (AIC) in the right hemisphere. TLE, Temporal lobe excision. Right, Sagittal section from the same MRI, showing damage in right hemisphere.

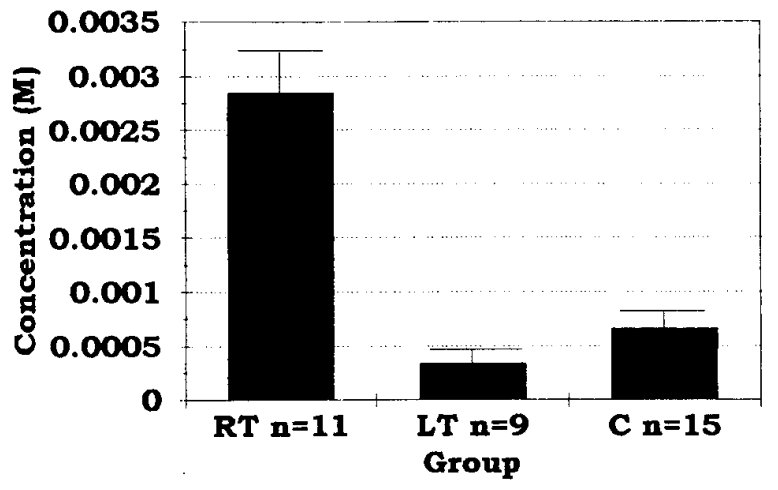

Figure 5. Mean recognition thresholds for the tastant citric acid. One patient from the RT group was unable to complete the recognition threshold assessment because of time constraints. $L T$, Left temporal; $R T$, right temporal; $C$, control.

ential rCBF was observed in the PGA. We believe that this probably reflects the mechanical stimulation of our delivery method, because cells responsive to somatosensory stimulation of the mouth are extensively interspersed within the PGA, and in fact probably represent a larger portion of the neural population than do taste-responsive cells (Yaxley et al., 1990). Any activity in this area attributable to citric acid stimulation was probably subtracted out in the analysis.

$\mathrm{rCBF}$ increases were observed bilaterally in the CLOF, which may represent the SGA described in the macaque monkey (Rolls et al., 1990), with activity in both the left hemisphere $(t=3.95$ at $x=-25, y=29, z=-18)$ and the right hemisphere $(t=3.25$ at $x=-25, y=24, z=-23)$. In contrast, a unilateral focus in the right anteromedial temporal lobe $(t=3.12$ at $x=17, y=1, z=$ -18 ) was observed (Fig. 6). Also favoring the right hemisphere, a strong focus of rCBF increase was observed in the right orbitofrontal cortex slightly medial to the area typically recognized as the SGA $(t=6.09$ at $x=17, y=37, z=-20)$ (Fig. 6).

\section{DISCUSSION}

In agreement with an earlier finding (Henkin et al., 1977), we found that surgical excision of the ATL leaves intact the ability to detect the presence of a sour stimulus, although it causes a deficit in recognition of the quality of that stimulus as sour. In contrast to this earlier report, however, which noted a deficit only in their LT group, we observed this dissociation preferentially among those patients who had an excision from the right ATL. It is possible that this discrepancy may have arisen because of the different methodologies used in the two studies to assess RTh. Henkin and co-workers asked their subjects to classify the solution as being different from water, with the choices being salt, bitter, sweet, or sour. In some cases, these words were presented in written form and placed in front of the subject. Perhaps the RT patients in the Henkin et al. (1977) study were aided by the verbal choice, whereas the LT patients were not. In the present study, no verbal cues were given and various responses were taken as correct (Table 2). Our RT group, therefore, did not have a verbal choice advantage over the LT group.

An elevated DTh was observed in one patient who, in addition to a right ATL excision, had removal of AIC (Fig. 4). Although this area does not correspond to the region of the anterior insular 


\section{Table 3. Significant foci of increased rCBF}

\begin{tabular}{rrrrl}
\multicolumn{1}{c}{$y$} & \multicolumn{1}{l}{$z$} & $t$ value & Brain area \\
\hline 17 & 37 & -20 & 6.09 & Right medial orbitofrontal cortex \\
28 & 48 & -9 & 4.96 & Right frontopolar cortex \\
9 & 41 & -27 & 4.66 & Right gyrus rectus \\
-21 & 36 & -12 & 4.32 & Left frontopolar cortex \\
-26 & 29 & -18 & 3.92 & Left caudolateral orbitofrontal cortex \\
-15 & 53 & -17 & 3.88 & Left gyrus rectus \\
-12 & -73 & 3 & 3.85 & Left lingual gyrus \\
32 & 25 & 24 & 3.72 & Right dorsolateral frontal cortex \\
-17 & 41 & -17 & 3.64 & Left medial orbitofrontal cortex \\
-25 & 24 & -23 & 3.25 & Right caudolateral orbitofrontal cortex \\
17 & 1 & -18 & 3.12 & Right anteromedial temporal lobe
\end{tabular}

The letters $x, y$, and $z$ refer to stereotaxic coordinates: $x$, medial-lateral position relative to midline (positive $=$ right); $y$, anterior-posterior position relative to the anterior commissure (positive $=$ anterior); $z$, superior-inferior position relative to the commissural line (positive $=$ superior). Foci were considered significant if their $t$ value was $>3.5$ or 3.0 for predicted peaks (Worsley et al., 1992).

cortex identified as PGA in nonhuman primates (Rolls et al., 1990), Penfield and Faulk (1955) reported eliciting disagreeable taste and gastric sensation from this region in human patients undergoing surgery for epilepsy. It could be that gustatory fibers passing from the PGA to the SGA were interrupted by surgery, which caused deficits in taste detection (Baylis et al., 1995).

The results from the PET study support and extend the results from the psychophysical study. We observed asymmetrical activation of the right anteromedial temporal lobe, as well as the right orbitofrontal cortex, at a site slightly medial to the secondary taste cortex (Fig. 4). This latter area is reciprocally connected to the amygdala (Turner et al., 1980) and has been implicated in stimulus-reinforcement learning, often involving food as a primary reinforcer (Kentridge et al., 1991). Therefore, the activity observed in these structures may reflect neural circuitry devoted to the limbic aspects of central gustatory processing, specifically the attachment of hedonic significance to a taste stimulus. Their asymmetrical activation is in accordance with our observation of gustatory recognition deficits after a right, but not left, ATL removal. It is likely that the surgical treatment received by our patients would entail damage to this region of the temporal lobe (Fig. 1). Therefore, we propose that the deficit in gustatory stimulus recognition may be attributable to disruption of functioning of this neural circuitry by the surgical procedure.

It is also possible that disruption of the pathway from the amygdala to the SGA (CLOF) could account for recognition deficits; however, bilateral activation of the CLOF ( $t=3.95$ on the left and $t=3.25$ on the right), corresponding to the area described as the SGA in the macaque (Rolls et al., 1990), was also observed (Fig. 6). Consequently, if the SGA is involved in taste quality discrimination, we should have observed RTh elevations in both left and right temporal resection groups.

The results from the present study are consistent with the nonhuman animal gustatory literature. Intensity-response functions derived from single-cell recording in the anterior insula/ frontal operculum of monkeys indicate that responses evoked as a function of concentration conform well to human psychophysical data (Scott et al., 1986b; Yaxley et al., 1990). For example, the lowest concentration that elicits a neural response corresponds well with the human DTh, suggesting that the PGA may be responsible for the conscious sensation of taste as well as for assessment of stimulus intensity. The elevated DTh observed in the patient who had AIC damage is in accordance with this hypothesis. Conversely, it is likely that processing in the PGA, undisturbed by the surgical procedures in all other patients, accounted for the normal DTh observed here and elsewhere after temporal lobectomy (Henkin et al., 1977).

Whether processing within the PGA is adequate to determine stimulus quality is a subject of current debate. Attempts to divide neurons in the PGA into discrete groups indicate that although it is possible to assign neurons to a small number of groups on the basis of their response profiles, the variability of responses within

Figure 6. Increased $\mathrm{rCBF}$ during citric acid stimulation. A horizontal slice through PET data superimposed on MRI scans averaged for all 10 subjects. Subtraction of the experimental condition from the control condition yielded the focal changes in CBF shown as a $t$ statistic. The range of $t$ values for the PET data (Table 3) are coded by the color scale. Significant foci of increased rCBF during presentation of citric acid in the left CLOF, right medial orbitofrontal cortex, and right anteromedial temporal lobe are illustrated (Table 3). The bilateral foci seen outside of the brain represent artifacts of masseter muscle activation attributable to mouth movement required to perform the task.

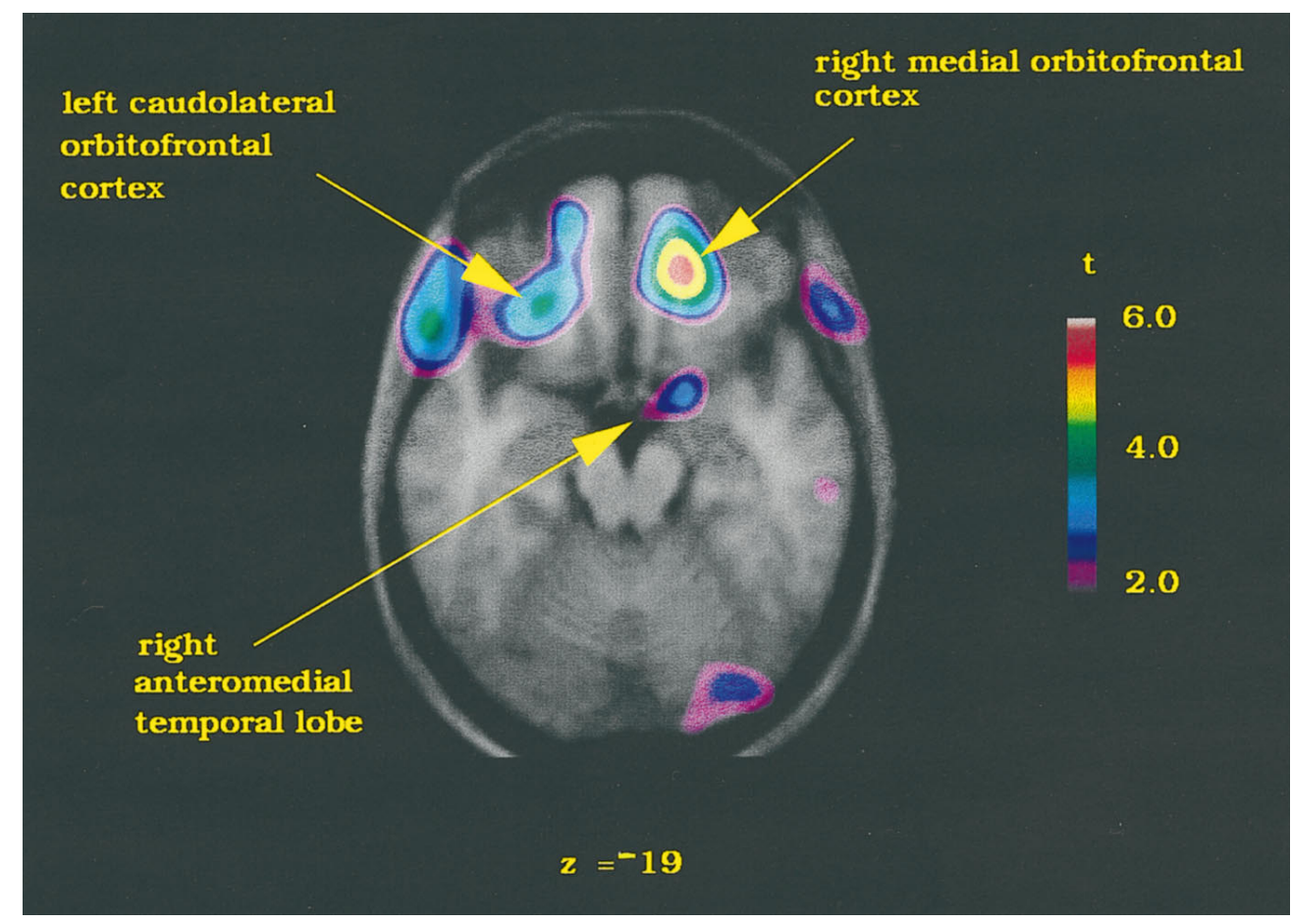


these groups is high (Scott et al., 1986b; Yaxley et al., 1990). Smith-Swintosky et al. (1991), however, evaluated the relationship between psychophysical studies of taste quality in the human, as reported by Kuznicki and Ashbaugh (1979) and Schiffman and Erickson (1971), and their own electrophysiological results of responses to taste quality in the alert macaque monkey. The correlation between their data and the data from Kuznicki and Ashbaugh (1979) was +0.91 , indicating a very close relationship between neural response evoked in the macaque PGA and the perceptual experience of taste quality in humans. When they performed the same analysis with the results from the Schiffman and Erickson study, however, the correlation $(+0.53)$ was not as high. Interestingly, Smith-Swintosky et al. (1991) suggest that this discrepancy arose because they used a higher concentration of salt, which was probably more aversive than the lesser concentration used in the psychophysical study. As a result, the response they recorded to salty stimuli was more akin to the response profiles elicited by aversive stimuli such as quinine. In fact, the correlation between the two data sets rose to +0.85 when they dropped the salty stimuli from the analysis. These results suggest that hedonic assessment may contribute to discrimination of taste quality; however, it is unlikely that hedonic processing is performed within the PGA, because lesions in this area do not disturb preference-aversion learning (for review, see Rolls, 1993).

Cells that respond to taste stimulation have been identified in the amygdala of the macaque monkey with use of single-cell recording techniques (Scott et al., 1993). Such neurons show no evidence of chemotopic arrangement, and they respond less selectively to the basic taste qualities than do neurons located at lower-order gustatory relays (Scott et al., 1993). Furthermore, responses across $1.5 \mathrm{log}$ units of stimulus concentration are nearly flat. Scott et al. (1993) have suggested therefore that taste-related activity in the amygdala does not provide an adequate basis for the discriminative capacity of humans or monkeys with regard to either stimulus quality or concentration. Rather, these authors suggest that the amygdala contributes to gustatory processes by "imparting hedonic appreciation and emotional significance to taste experience," as the amygdala has long been implicated in hedonic processing (Jones and Mishkin, 1972; Ono et al., 1983; LeDoux, 1987). The amygdala has also been implicated in assessment of novel flavors (Dunn and Everitt, 1988; Rolls and Rolls, 1973; Borsini and Rolls, 1984) and in making cross-modal associations between a previously neutral stimulus and a primary reinforcing stimulus (such as the taste of food) (Gaffan and Harrison, 1987; Gaffan et al., 1988; Kentridge et al., 1991; Rolls, 1993). Additionally, an intact amygdala is critical for the expression of CTAs (Aggleton et al., 1981; Yamamoto et al., 1994).

Clearly, the nonhuman animal literature also suggests a dissociation of gustatory functions, with the PGA coding for taste detection and intensity and the amygdala coding for the hedonic valence of a taste stimulus. Perhaps with the integration of processing within both the PGA and the amygdala, the ability to recognize taste quality emerges. As such, sensation of a stimulus and assessment of its intensity are computed in the PGA, and the basic "outline" of stimulus quality is established, evidenced by the ability to identify discrete groups of gustatory neurons (Scott et al., 1986b; Yaxley et al., 1990). A gustatory code containing this information is then sent to the amygdala, where it is modulated on the basis of previous associations and biological implications of the stimulus before the code is relayed to successive areas involved in taste and ingestive processing. Reciprocal connections between the amygdala and the PGA (Turner et al., 1980), the orbitofrontal cortex, including SGA (Amaral and Price, 1984, Wiggins et al., 1987), and the subcortical solitary tract gustatory nucleus (Norgren, 1974; Price, 1981) have been demonstrated in nonhuman animals. Destruction of the amygdala leads to loss of fibers of passage (Dunn and Everitt, 1988). Therefore, resection of the amygdala and/or these associated gustatory pathways could lead to difficulties in determining the nature of a stimulus quality and consequently lead to the elevated RTh observed here.

In conclusion, on the basis of these results and reports in the literature, we postulate that human taste sensation occurs in the PGA, located in the anterior insula/frontal operculum, whereas taste recognition involves integration of the gustatory code with motivational and hedonic networks related to feeding, which we suggest occurs in the anteromedial temporal lobe. Finally, our results suggest that gustatory processing at the level of the ATL, at least for citric acid, occurs preferentially in the right hemisphere.

\section{REFERENCES}

Aggleton JP, Petrides M, Iversen SD (1981) Differential effects of amygdaloid lesions on conditioned taste aversion learning by rats. Physiol Behav 27:397-400.

Amaral DG, Price JL (1984) Amygdalo-cortical projections in the monkey (Macaca fascicularis). J Comp Neurol 230:465-496.

Baylis LL, Gaffan D (1991) Amygdalectomy and ventromedial prefrontal ablation produce similar deficits in food choice and in simple object discrimination learning for an unseen reward. Exp Brain Res 86:617-622.

Baylis LL, Rolls ET, Baylis GC (1995) Afferent connections of the caudolateral orbitofrontal cortex taste area of the primate. J Neurosci 64:801-812.

Benjamin RM, Burton H (1968) Projection of taste nerve afferents to anterior opercular-insular cortex in squirrel monkey (Saimiri sciureus). Brain Res 7:208-220.

Blum J, Chow K, Pribram K (1950) A behavioral analysis of the organization of the parieto-temporo-preoccipital cortex. J Comp Neurol 93:53-100

Borsini F, Rolls ET (1984) Role of noradrenaline and serotonin in the basolateral region of the amygdala in food preferences and learned taste aversions in the rat. Physiol Behav 33:37-43.

Cerf B, Faurion A, MacLeod P, Van de Moortele P-F, LeBihan D (1996) Functional MRI study of human gustatory cortex. NeuroImage 3:S342.

Contreras R, Frank M (1979) Sodium deprivation alters neural responses to gustatory stimuli. J Gen Physiol 73:569-594.

Doty RL, Shaman P, Dann M (1984) Development of the University of Pennsylvania smell identification test: a standardized microencapsulated test of olfactory function. Physiol Behav 32:489-502.

Dunn LT, Everitt BJ (1988) Double dissociations of the effects of amygdala and insular cortex lesions on conditioned taste aversion, passive avoidance, and neophobia in the rat using the excitotoxin ibotenic acid. Behav Neurosci 102:3-23.

Evans AC, Thompson CJ, Marrett S, Meyer E, Mazza M (1991a) Performance evaluation of the PC-2048: a new 15-slice encoded-crystal PET scanner for neurological studies. IEEE Trans Med Imaging 10:90-98.

Evans AC, Marrett S, Torrescorzo J, Ku S, Collins L (1991b) MRI-PET correlation in three dimensions using a volume-of-interest (VOI) atlas. J Cereb Blood Flow Metab 12:A69-A78.

Evans AC, Marrett S, Neelin P, Collins L, Worsley K, Dai W, Milot S, Meyer E, Bub D (1992) Anatomical mapping of functional activation in stereotactic coordinate space. NeuroImage 1:43-53.

Gaffan D, Harrison S (1987) Amygdalectomy and dissociation in visual learning for auditory secondary reinforcement by monkeys. J Neurosci 7:2285-2292.

Gaffan EA, Gaffan D, Harrison S (1988) Visual-visual associative learning and reward-association learning in monkeys: role for the amygdala. J Neurosci 9:558-569. 
Henkin R, Comiter H, Fedio P, O’Doherty D (1977) Defects in taste and smell recognition following temporal lobectomy. Trans Am Neurol Assoc 102:146-150.

Hirsch J, DeLaPaz R, Relkin N, Victor J, Bartoshuk L, Norgren R, Pritchard T (1994) Localization of human gustatory cortex using functional magnetic imaging. Paper presented at the meeting for the Association of Chemoreception Sciences, Sarasota, FL.

Jacobs KM, Mark GP, Scott TR (1988) Taste responses in the nucleus tractus solitarius of sodium-deprived rats. J Physiol (Lond) 406:393-410.

Jones EG, Mishkin M (1972) Limbic lesions and the problem of stimulus-reinforcement associations. Exp Neurol 36:362-377.

Kentridge RW, Shaw C, Aggleton JP (1991) Amygdaloid lesions and stimulus-reward associations in the rat. Behav Brain Res 42:57-66.

Kluver H, Bucy P (1938) An analysis of certain effects of bilateral temporal lobectomy in the rhesus monkey, with special reference to "psychic blindness." J Psychol 5:33-54.

Kuznicki JT, Ashbaugh N (1979) Taste quality differences within sweet and salty taste categories. Sens Proc 3:157-182.

LeDoux JE (1987) Emotion. In: Handbook of physiology. The nervous system (Plum F, ed), pp 419-459. Bethesda, MD: American Physiological Society.

Norgren R (1974) Gustatory afferents to the ventral forebrain. Brain Res 81:285-295.

Ono T, Fukuda M, Nishino H, Sasaki K, Muramoto K-I (1983) Amygdaloid neuronal responses to complex visual stimuli in an operant feeding situation in the monkey. Brain Res Bull 11:515-518.

Penfield W, Faulk ME (1955) The insula. Brain 78:445-470.

Petrides M, Alivisatos B, Pandya DN, Evans AC (1996) Gustatory cortex: comparative architectonic analysis in the human and the macaque brain and functional data. NeuroImage 3:S344.

Pribram K, Bagshaw M (1954) Further analysis of the temporal lobe syndrome utilizing frontotemporal ablations. J Comp Neurol 98:347-373.

Price JL (1981) The efferent projections of the amygdaloid complex in the rat, cat and monkey. In: The amygdaloid complex (Ben-Ari Y, ed), pp 121-132. Amsterdam: Elsevier.

Raichle ME, Martin WRW, Herscovitch P, Mintum MA, Markham J (1983) Brain blood flow measured with intravenous $\mathrm{H}_{2} \mathrm{O}^{15}$. II. Implementation and validation. J Nucl Med 24:790-798.

Rolls BJ, Rolls ET (1973) Effects of lesions in the basolateral amygdala on fluid intake in the rat. J Comp Physiol Psych 83:240-247.

Rolls ET (1993) The neural control of feeding in primates. In: Neurophysiology of ingestion (Booth DA, ed), pp 137-169. Oxford: Pergamon.

Rolls ET, Baylis LL (1994) Gustatory, olfactory and visual convergence within the primate orbitofrontal cortex. J Neurosci 14:5437-5452.

Rolls ET, Scott TR, Sienkiewicz ZJ, Yaxley S (1988) The responsiveness of neurons in the frontal opercular gustatory cortex of the macaque monkey is independent of hunger. J Physiol (Lond) 397:1-12.

Rolls ET, Sienkiewicz ZJ, Yaxley S (1989) Hunger modulates the responses to gustatory stimuli of single neurons in the caudolateral orbitofrontal cortex of the macaque monkey. Eur J Neurosci 1:53-70.

Rolls ET, Yaxley S, Sienkiewicz ZJ (1990) Gustatory responses of single neurons in the orbitofrontal cortex of the macaque monkey. J Neurophysiol 64:1055-1066.

Schiffman SS, Erickson RP (1971) A psychophysical model for gustatory quality of sodium salts. Physiol Behav 7:617-633.

Scott TR (1992) Taste, feeding, and pleasure. In: Progress in psychobiology and physiological psychology, Vol 15 (Epstein AN, Morrison AR, eds), pp 231-291. San Diego: Academic.

Scott TR, Yaxley S, Sienkiewicz ZJ, Rolls ET (1986a) Gustatory responses in the nucleus tractus solitarius of the alert cynomolgus monkey. J Neurophysiol 55:182-200.

Scott TR, Yaxley S, Sienkiewicz ZJ, Rolls ET (1986b) Gustatory responses in the frontal opercular cortex of the alert cynomolgus monkey. J Neurophysiol 56:876-890.

Scott TR, Zoltan K, Oomura Y, Nishino H, Plata-Salaman CR, Lenard L, Giza BK, Aou S (1993) Gustatory neural coding in the amygdala of the alert macaque monkey. J Neurophysiol 69:1810-1820.

Small DM, Zatorre RJ, Jones-Gotman M, Petrides M, Evans AC (1996) Flavour processing: more or less than the sum of its parts? NeuroImage 3:S345.

Smith-Swintosky VL, Plata-Salaman CR, Scott TR (1991) Gustatory neural coding in the monkey cortex: stimulus quality. J Neurophysiol 66:1156-1165.

Talairach J, Tournoux P (1988) Co-planar stereotaxic atlas of the human brain. New York: Thieme.

Turner BH, Mishkin M, Knapp M (1980) Organization of the amygdalopetal projections from modality-specific cortical association areas in the monkey. J Comp Neurol 191:515-543.

Wechsler D (1981) Wechsler adult intelligence scale-revised. New York: The Psychological Corporation.

Wiggins LL, Rolls ET, Baylis GC (1987) Afferent connections of the orbitofrontal cortex taste area of the primate. Chem Sens 12:206.

Worsley KJ, Evans AC, Marrett S, Neelin P (1992) A three-dimensional statistical analysis for CBF activation studies in human brain. J Cereb Blood Flow Metab 12:900-918.

Yamamoto T, Shimura T, Sako N, Yasoshima Y, Sakai N (1994) Neural substrates for conditioned taste aversion in the rat. Behav Brain Res 65:123-137.

Yaxley S, Rolls ET, Sienkiewicz ZJ (1990) Gustatory responses of single neurons in the insula of the macaque monkey. J Neurophysiol 63:689-700. 